Nadja Milewski

\title{
Erwerbsbeteiligung und Einstellungen zur Familie von türkischen Migrantinnen im Generationenvergleich
}

\author{
Labour force participation and family attitudes of first and second \\ generation Turkish migrant women
}

\begin{abstract}
Zusammenfassung:
Die Arbeitsmarktbeteiligung von Frauen mit türkischem Migrationshintergrund in Deutschland ist geringer als die anderer Migrantengruppen und als die von Frauen ohne Migrationshintergrund. Diese Studie untersucht mit Daten des Generations and Gender Survey (2005/2006), inwiefern sich Frauen der ersten und zweiten Migrantengenerationen hinsichtlich ihrer Erwerbsbeteiligung unterscheiden und welche Faktoren dafür ursächlich sind. In Einklang mit der Humankapitaltheorie lässt sich für die zweite Generation eine stark gesteigerte Erwerbsbeteiligung feststellen: Während in der ersten Generation nur etwa 34 Prozent der Frauen einer Beschäftigung nachgehen, beteiligen sich rund 63 Prozent in der zweiten Generation am Arbeitsmarkt. Besondere Berücksichtigung erfährt der Sozialkapitalansatz bzw. Aspekte intergenerationaler Transmission und Solidarität. Demnach übt - neben Bildung und Familienstand - eine Erwerbstätigkeit der Mutter einen Einfluss auf die Erwerbsbeteiligung der Tochter aus, und stärker egalitäre Genderrolleneinstellungen begünstigen eine Erwerbstätigkeit.
\end{abstract}

Schlagworte: Erwerbsbeteiligung, türkische Migrantinnen, erste und zweite Migrantengeneration, intergenerationale Transmission, Generations and Gender Survey Deutschland

\begin{abstract}
:
The extent of labour force participation of women with a Turkish migration background in Germany is lower than that of women of other ethnic origins or that of non-migrant women. In this study, we focus on a within-group comparison of the labour force participation among Turkish women. Using data of the Generations and Gender Survey (2005/ 2006), we distinguish between first and second immigrant generations. The study pays special attention to both the human and social capital approach and aspects of intergenerational transmission and solidarity between generations. In line with the human capital theory, a strong increase in labour force participation can be noted among women belonging to the second migrant generation: Whereas only about 34 per cent of first generation immigrants participate in the workforce, the share among the second generation amounts to about 60 per cent. In addition to family status and education, mothers' employment is found to increase the likelihood of the daughter's participation in the workforce. Also, more egalitarian gender role attitudes favour a higher degree of employment.
\end{abstract}

Key words: Labour force participation, female Turkish immigrants, first and second immigrant generation, intergenerational transmission, German Generations and Gender Survey 


\section{Einleitung}

Ungleichheiten im Bildungssystem und auf dem Arbeitsmarkt ist - in Deutschland wie international - die meiste Aufmerksamkeit in der Forschung zur Integration von Migranten gewidmet worden (siehe Alba et al. 1994; Nauck 2011). Dabei richtet sich das Forschungsinteresse vor allem auf ethnische Ungleichheiten bzw. Unterschiede zwischen Personen mit und ohne Migrationshintergrund. Relativ jung sind hingegen Untersuchungen zu Integrationsprozessen innerhalb der zugewanderten Bevölkerung. Das Forschungsinteresse der vorliegenden Untersuchung richtet sich auf Dynamiken innerhalb der größten in Deutschland lebenden Migrantengruppe und fragt nach den Determinanten der Erwerbsbeteiligung von Frauen mit türkischem Migrationshintergrund.

Sowohl im Vergleich mit anderen Zuwanderergruppen als auch mit Personen ohne Migrationshintergrund zeigen Personen mit türkischem Migrationshintergrund in Studien zu Bildungsbeteiligung und -erfolg sowie zur Arbeitsmarktintegration die niedrigsten Werte, und dieses unterdurchschnittliche Abschneiden setzt sich - trotz leichter Aufwärtsmobilität gegenüber der Elterngeneration - unter den Nachkommen der ersten Migrantengeneration fort (z.B. Fincke 2009). So sieht Kalter (2008: 327) die türkische zweite Generation in einer "Sonderrolle" unter den klassischen Arbeitsmigranten in Deutschland und wirft die „Frage einer eventuell dauerhaften (im Sinne von Generationen) Verfestigung ethnischer Ungleichheiten" auf.

Bei der Frage nach der sozialen Mobilität der zweiten Migrantengeneration erscheint die Rolle der Familie gerade in Migrantengruppen, die aus Ländern mit patriarchalischer Familientradition kommen, zugleich zentral und widersprüchlich. Nauck (2004) sieht eine familialistische Orientierung bei Arbeitsmigranten als Hemmnis für eine Statusmobilität der zweiten Generation, wenn alle sozialen Kontakte innerhalb des Verwandtschaftsnetzes und nicht mit Mitgliedern der Mehrheitsgesellschaft stattfinden. Gleichzeitig dient die Familie als Voraussetzung für eine soziale Eingliederung, wenn sie Unterstützung in Form von sozialem, kulturellem oder ökonomischen Kapital für Integrationsprozesse bereitstellt.

Während sich bei Männern Nachteile von Migranten in der Arbeitsmarktintegration zum Beispiel im Risiko der Arbeitslosigkeit, im Arbeitsmarktsektor und im Einkommen zeigen, scheint für Frauen die Frage der Erwerbsbeteiligung per se entscheidend (Algan et al. 2010; Höhne/Koopmans 2010). Ihre Arbeitsmarktbeteiligung ist ein Indikator für die strukturelle Inkorporation von Migranten. Gleichzeitig reflektiert sie kulturelle Transformationsprozesse, da die Berufstätigkeit der Frau von kulturellen und/oder religiösen Normen hinsichtlich der Rolle der Frau in der Familie, Traditionen der Erwerbsbeteiligung in den Herkunftsländern der Migrantinnen wie auch Präferenzen für Familiengröße und -struktur abhängig ist (Reimers 1985; Antecol 2000; Blau et al. 2012). So fand Reimers (1985 für die USA), dass die Variation in der Arbeitsmarktbeteiligung von Frauen verschiedener Zuwanderergruppen größer ist als jene z.B. in der Zahl der Arbeitsstunden der Erwerbstätigen, und führt dies auf kulturelle Aspekte zurück.

Die Relevanz kultureller Normen hinsichtlich der Frauenerwerbsbeteiligung in Deutschland ergibt sich daraus, dass ein großer Teil der etwa 16 Millionen Personen mit Migrationshintergrund, die heute in Deutschland leben (bzw. deren Eltern), aus Ländern mit affinalverwandtschaftlichem, insbesondere patrilinearem Familiensystem stammt, in denen sehr viel stärker als in Deutschland die Geschlechterarbeitsteilung und die Fürsorge der Generationen füreinander normativ verankert sind (Baykara-Krumme et al. 2011). Blau 
et al. (2012) betonen die Bedeutung der intergenerationalen Transmission für die Arbeitsmarktintegration von Migranten: Wenn Mütter ihren Kindern Geschlechterrollen vermitteln, die anders sind als jene im Zielland, könnte dies bei einem steigenden Anteil von Migranten auf Bevölkerungsebene zu substanziellen Veränderungen der Frauenerwerbstätigkeit und der Fertilität der Bevölkerung eines Landes führen. Zudem sind Langzeitfolgen von permanenter oder phasenweiser Nichterwerbstätigkeit in einem Land, in dem die Altersabsicherung über ein auf Erwerbstätigkeit basierendes Rentensystem erfolgt, erheblich. Obwohl der Anteil der älteren Menschen mit Migrationshintergrund mit knapp zehn Prozent gegenwärtig noch nicht einmal halb so groß ist wie der unter der Bevölkerung ohne Migrationshintergrund, deutet sich schon heute eine schlechtere finanzielle Lage der Rentner mit Migrationshintergrund an. So sind die relativ niedrigen Renten von Zuwanderern nur zum Teil mit niedrigen Bildungsabschlüssen zu erklären, sondern gehen auch mit relativ langen Zeiten in Arbeitslosigkeit bzw. Nichterwerbstätigkeit einher (Tucci/Yildiz 2012).

Vor diesem Hintergrund erscheint die Frage nach der intergenerationalen Mobilität wichtig: Wie wird sich die Berufstätigkeit in der zweiten Migrantengeneration entwickeln? Nauck (2011: 86) konstatiert einerseits, dass es „für die Optimierung des physischen Wohlbefindens in der deutschen Gesellschaft kaum Alternativen zur Erwerbsarbeit gibt und Migrantenfamilien somit ,keine andere Wahl' haben, als in die Humankapitalausstattung ihrer Kinder zu investieren“". Andererseits macht Soremski (2008) auf die Perspektive der Frauen aufmerksam. Gegenstand von Transmissionsprozessen in Familien mit türkischem Migrationshintergrund seien vor allem familialistische und normative Geschlechterrollenorientierungen, die die Frau weniger in der außerhäuslichen Berufstätigkeit als vielmehr in der Familienarbeit sehen. Daher stelle sich die Frage, welche „Autonomiespielräume der zweiten Generation gewährt werden, sich im Verhältnis zu familialen Erwartungen und gesellschaftlichen Anforderungen zu positionieren" (Soremski 2008: 238) angesichts einer umfassenden strukturellen Benachteiligung im schulischen und beruflichen Bereich.

Diese Studie stellt den Generationenvergleich in den Mittelpunkt und untersucht mit Daten des Generations and Gender Survey (2005/06) die Erwerbsbeteiligung von Frauen mit türkischem Migrationshintergrund. Besonderes Augenmerk liegt auf den Generationenbeziehungen und der intergenerationalen Transmission als Einflussfaktoren.

\section{Untersuchungsansätze}

Zur Erklärung von Inkorporationsprozessen von Immigranten der ersten Generation auf dem Arbeitsmarkt ist vor allem aus dem Humankapitalansatz die Arbeitsmarktassimilationstheorie (Chiswick 1978) herangezogen worden: Die meisten Studien finden, dass Immigranten - je nach Herkunfts- und Zielland - anfangs ökonomische Nachteile im Vergleich zu Nichtmigranten haben, diese Nachteile sich aber mit steigender Aufenthaltsdauer verringern bzw. nivellieren. Die Nachteile der ersten Generation lassen sich dabei vor allem auf eine Entwertung des Humankapitals (Bildungszertifikate), eine negative Selektion der Migranten sowie auf Präferenzen und Motive der Zuwanderer für eine Rückwanderung bzw. eine damit in Zusammenhang stehende Bereitschaft, in aufnahmelandspezifisches Wissen zu investieren, zurückführen (vgl. Kalter 2008, van Tubergen 2008). 
Der Großteil der Forschung zur ökonomischen Eingliederung von Immigranten hat sich bisher mit Männern auf dem Arbeitsmarkt beschäftigt. Im Fokus standen vor allem Löhne, Sektoren und Beschäftigungsraten bzw. Arbeitslosigkeit. Zur Erklärung unterschiedlicher Erträge auf dem Arbeitsmarkt wurden auch Theorien zur Diskriminierung, Theorien zur Segmentation des Arbeitsmarktes und die Rolle spezifischer Ressourcen vorgebracht (siehe Kalter 2008).

Für Frauen hat sich die Forschung bisher vor allem auf die englischsprachigen Einwanderungsländer konzentriert. Erst in den letzten Jahren wurde Europa mit seiner zunehmenden Rolle als Immigrationsregion, seiner Vielzahl an Herkunftsländern von Migranten und seinen unterschiedlichen Immigrations- und Integrationspolitiken für Studien zur Arbeitsmarktpartizipation von Frauen genutzt (Rendall et al. 2010). Die Ergebnisse für die Arbeitsmarktbeteiligung in west- und südeuropäischen Zielländern stehen in Einklang mit der Theorie zur Arbeitsmarktassimilation: Die Arbeitsmarktbeteiligung von Migrantinnen beginnt auf niedrigem Niveau und nähert sich mit steigender Aufenthaltsdauer in etwa dem Niveau der Nichtmigrantinnen an (Rendall et al. 2010).

Zum Erfolg von Migrantinnen auf dem Arbeitsmarkt selbst lassen sich die Ergebnisse jedoch nicht mit der Theorie der Arbeitsmarktassimilation erklären, sondern eher mit der Haushaltspezialisierungstheorie (Becker 1991). So zeigte Long (1980), dass bei Immigrantinnen in den USA das Einkommen mit zunehmender Aufenthaltsdauer sinkt, und führte dies auf einen Partnereffekt zurück: Demnach würden Frauen vor allem zu Beginn nach der Migration verstärkt auf dem Arbeitsmarkt aktiv und relativ hohe Löhne erzielen, um ihrem Partner in dieser Zeit zu ermöglichen, sich ziellandspezifisches Humankapital anzueignen. Im Laufe der Zeit würde sich die Position des Partners dann auf dem Arbeitsmarkt verbessern und die Frau ihre Erwerbstätigkeit reduzieren. Diese Hypothese wurde auch als Familieninvestitionshypothese (Duleep 1998) bezeichnet.

Im Kontrast dazu stehen die Forschungsergebnisse von Soremski (2008) zur sozialen Mobilität von Personen mit türkischem Migrationshintergrund in Deutschland. Im Sinne Bourdieus (1983) stellt sie in einer qualitativen Studie die Rolle von kulturellem Kapital in sozialen Transformationsprozessen heraus: Für Frauen aus der Türkei habe sich mit der Arbeitsmigration des Mannes eine soziale Neuorientierung eröffnet, die sich auf den Außenraum der Familie richtet. Eine außerhäusliche Berufstätigkeit der Frau stelle keinen Bruch mit der Haufrauen- und Mutterrolle dar, sondern übertrage familiale Motive vom häuslichen Bereich in den Arbeitskontext, wobei der Ehemann der Haupternährer der Familie bleibe. Soremski (2008: 246): „Die soziale Logik weicht einer wertrationalen Logik, die auf einen sozialen Statusaufstieg der nachfolgenden Generation als auch der eigenen Person bezogen ist"،. Demnach findet im Migrationskontext eine soziale Transformation geschlechtsspezifischer Traditionen statt, so dass eine Berufstätigkeit der Frau nicht im Widerspruch zur sozialen Ordnung steht - sie kann Bestandteil der Geschlechterrolle werden, welche dann als solche auch an die Folgegeneration tradiert wird.

$\mathrm{Zu}$ ähnlichen Überlegungen gelangt Nauck (2011) hinsichtlich sozialen Kapitals in Migrantenfamilien, indem er Sozialkapitaltheorien in soziale Produktionsfunktionen integriert. Demnach würden sich die Anfangsinvestitionen einer Migration erst in der Folgegeneration durch - auch ökonomische - Renditen rechtfertigen. Erwerbstätigkeit erscheine in Deutschland als nicht substituierbares Gut, das entweder komplementär oder konkurrierend $\mathrm{zu}$ anderen Handlungsoptionen stehe. Demnach würde eine Investition in Humankapital dann erfolgen, ,wenn sozialer Status in der Mehrheitsgesellschaft gesucht wird und somit über Assimilation das Handlungsziel der sozialen Anerkennung angestrebt 
wird“ (Nauck 2011: 86). Umgekehrt ständen eine Aufrechterhaltung von strong ties und soziale Anerkennung über Segmentation miteinander in Zusammenhang.

Damit rückt die Rolle der Familie verstärkt in das Interesse der Bildungs- und Arbeitsmarktforschung. In bisherigen Studien zur Arbeitsmarktintegration von Migranten wurden meist weak ties in den Mittelpunkt gestellt (Granovetter 1973; van Tubergen 2008). Dabei hängt Sozialkapital von der Anzahl der sozialen Kontakte, deren Unterstützungswillen und -ressourcen ab, und soziale Kontakte können bei der Suche nach Arbeit bzw. bei der Aneignung von Kompetenzen helfen (de Graaf/Flap 1988). Jüngere Forschung hat verstärkt den Effekt von strong ties auf die Erwerbstätigkeit untersucht und sich dabei auf die Rolle des Partners konzentriert (Lin 1999; van Tubergen 2008). Die Unterstützungsleistung durch den Partner auf die Erwerbsposition seiner Frau ist abhängig von dessen Humankapital: Höheres Humankapital des Partners - Bildung und Erwerbstätigkeit - ist mit einer höheren Arbeitsmarktbeteiligung und -erfolg der Frau assoziiert (van Tubergen 2008).

Strong ties umfassen jedoch nicht nur den Lebenspartner, sondern zeigen sich insbesondere in den Generationenbeziehungen. So sind die Regionen Europas, die durch strong family ties bzw. ein Deszendenzfamilienregime gekennzeichnet sind, also vor allem die Mittelmeerländer, durch eine hohe intergenerationale Solidarität, etwa in der Kinderbetreuung und der Altenpflege, materielle Transferleistungen innerhalb der Familie und eine Arbeitsteilung der Geschlechter mit dem Mann in der Versorgerrolle und der Frau in der Fürsorgerolle, gekennzeichnet (Reher 1998; Nauck/Suckow 2006; Kal$\mathrm{mijn} /$ Saraceno 2008).

Je enger intrafamiliäre Aufgaben und Erwerbstätigkeit verbunden sind, desto geringer sind die Opportunitätskosten für Generationenbeziehungen (Becker 1991). Folglich steht eine außerhäusliche Berufstätigkeit der Frau in Konkurrenz zur intergenerationalen Pflege. Auf der Makroebene zeigt sich im europäischen Vergleich, dass Länder, die eher eine Gleichberechtigung der Geschlechter unterstützen, auch eine höhere Frauenerwerbstätigkeit haben. In Ländern mit strong family ties, geschlechtsspezifischer Arbeitsteilung und hoher Verantwortung der Kinder für ihre alternden Eltern ist die Frauenerwerbsbeteiligung relativ niedrig (Uunk et al. 2005).

Umgekehrt ist eine Unterstützungsleistung, die von der älteren zur jüngeren Generation geleistet werden kann, die Betreuung von Enkelkindern: Wheelock/Jones (2002, für England) und Hank/Buber (2007) zeigten, dass die Unterstützung durch die Familie von Frauen als essenzielle Bedingung gesehen wird, um nach der Geburt von Kindern in den Arbeitsmarkt zurückzukehren; Großeltern werden dabei als beste Lösung für Kinderbetreuung gesehen und können die Kosten der Erwerbstätigkeit senken.

Blau et al. (2012) zeigten auch, dass kulturelle Muster des Bildungserwerbs und der Erwerbstätigkeit zum Teil auf intergenerationale Transmission von Geschlechterrollen zurückzuführen sind, wobei der Einfluss der Mutter auf Frauen größer ist als der des Vaters. Auch Soremski (2008) stellt die Erwerbsorientierung der Mutter als zentralen Determinanten der sozialen Mobilitätsorientierung in der zweiten Migrantengeneration heraus (vgl. Boos-Nünning/Karakaşoğlu 2005, Kawaguchi/Miyazaki 2009). 


\section{Kontext}

Immigrantinnen aus der Türkei kommen aus einem Land, in dem die Erwerbsbeteiligung von Frauen unter 40 Prozent liegt, was selbst im Vergleich mit anderen Ländern mit patrilinearen Familienstrukturen eine geringe Rate ist (Nauck/Suckow 2006). Dabei ist die Erwerbsbeteiligung unter Müttern niedriger; etwa zwei Drittel der Mütter sind Hausfrauen, unter den kinderlosen Frauen knapp die Hälfte (Hancioglu/ Ergöcmen 2004), wobei sich die verschiedenen Regionen der Türkei stark unterscheiden (El-Menouar/ Fritz 2009).

In Bezug auf die Eingliederung von Migrantinnen und Migranten aus der Türkei in Deutschland fällt auf, dass diese unterdurchschnittliche Bildungsabschlüsse und Arbeitsmarktpositionen erreichen sowie längere Zeiten in Arbeitslosigkeit verbringen im Vergleich zu Personen ohne Migrationshintergrund, aber auch relativ zu Migranten aus anderen Ländern (Granato 2003; Constant et al. 2006, Hans 2010; Höhne/Koopmans 2010; Tucci 2011; Burkert et al. 2012). Frauen mit türkischem Migrationshintergrund haben von allen Zuwandergruppen mit etwa 50 Prozent die geringste Erwerbsbeteiligung (Stichs 2008; Fincke 2009; für Österreich: Neuwirth/Wernhart 2007). Dies spiegelt sich auch in den Geschlechterrolleneinstellungen junger Erwachsener in Deutschland wider: von Below/Karakoyun (2007) fanden unter türkischen Frauen eine deutlich geringere Befürwortung egalitärer Arbeitsteilung als unter Deutschen und Italienerinnen.

Unter Zuwanderern aus der Türkei ist mit zunehmender Aufenthaltsdauer bzw. in der zweiten Migrantengeneration eine Entwicklung hin zu höherer Bildungsbeteiligung und höherer Arbeitsmarktintegration im Vergleich zur ersten Generation zu erkennen. Dennoch ist nur in etwa die Hälfte der Frauen der zweiten türkischen Generation auf dem Arbeitsmarkt aktiv (Seifert 1997; Granato 2003; Kalter 2008; Fincke 2009; Algan et al. 2010; Sürig/Wilmes 2011). Ähnliche Befunde gibt es auch für andere Zielländer der ehemaligen Arbeitsmigranten und ihre Nachkommen aus der Türkei (Biffl 2002, für Österreich; Heering/ter Bekke 2008, für die Niederlande) bzw. für Migrantinnen aus muslimischen Ländern (Fleischmann/Dronkers 2010).

In Zusammenhang mit höherer Bildung und Erwerbstätigkeit von Migrantinnen wurde in der Forschung zu den Generationenbeziehungen vor allem die funktionale Solidarität, also der Austausch von Unterstützungsleistungen, diskutiert. Verschiedentlich wurde gezeigt, dass die steigende Bildung und Erwerbsbeteiligung der Frau in europäischen Ländern mit größerer räumlicher Distanz und entsprechend sinkender Kontakthäufigkeit zwischen den Generationen einhergeht (u.a. Höpflinger 1993; Kalmijn 2006). Für Deutschland wurde jedoch demonstriert, dass sich die Generationen eher nicht durch die Migration entfremden (Baykara-Krumme et al. 2011). Einerseits äußern vor allem ältere Migranten aus der Türkei - in Einklang mit der Value-of-children-Theorie - wenn auch nicht einseitige, so doch relativ hohe ökonomisch-utilitaristische Erwartungen an ihre Kinder, vor allem an die Töchter (Olbermann 2003; Zimmermann 2012). Andererseits zeigt sich auch in der zweiten Generation mit türkischem Hintergrund der Wille, die Elterngeneration zu unterstützen (Carnein/Baykara-Krumme in diesem Heft). Angesichts der Persistenz der Normen zur intergenerationalen Solidarität und der Konkurrenz zwischen außerhäuslicher und Familienarbeit ist zu fragen, inwiefern sich Einstellungen zu intergenerationalen Unterstützungsleistungen auf die Erwerbsbeteiligung von Frauen auswirken. 


\section{Hypothesen}

Ausgehend von diesen Überlegungen werden fünf zentrale Hypothesen zur Erwerbsbeteiligung von Frauen abgeleitet:

H1) Arbeitsmarktassimilationshypothese der Generationen: Nach der Humankapitaltheorie ist eine stärkere Arbeitsmarktbeteiligung von Migrantinnen der zweiten gegenüber der ersten Generation zu erwarten. Eine höhere Bildung ist mit einer höheren Erwerbsbeteiligung assoziiert (Becker 1991). In der zweiten Generation liegt allgemein eine höhere Bildungsbeteiligung vor als in der ersten (Söhn 2011), und diese ist auch stärker ziellandspezifisch, da der Schulbesuch in Deutschland stattfand und entsprechend Sprachkenntnisse vorliegen. Somit sollte eine Entwertung des Humankapitals weniger eine Rolle spielen (Fincke 2009; van Tubergen 2006).

H2) Hypothese der intergenerationalen Transmission: Die Erwerbsbeteiligung einer Frau ist höher, wenn ihre Mutter bereits erwerbstätig war (Soremski 2011; Blau 2012).

H3) Hypothesen zur intergenerationalen Unterstützung: Je stärker eine Frau die Normen der intergenerationalen Unterstützungsverpflichtung vertritt, desto geringer ist die Erwerbsbeteiligung (Becker 1991).

H4) Geschlechterrollenhypothese: Frauen, die eine geschlechterspezifische Arbeitsteilung präferieren, haben eine geringere Erwerbsbeteiligung als jene, die eine eher egalitäre Rollenverteilung befürworten (Kawaguchi/Miyazaki 2009).

H5) Kompositionshypothese: Etwaige Unterschiede in der Erwerbsbeteiligung der beiden Migrantengenerationen sollten sich verringern, wenn man weitere Merkmale der sozio-demografischen Komposition der Gruppen berücksichtigt. Für die Variable Staatsbürgerschaft wird angenommen, dass eine deutsche Nationalität mit einer höheren Erwerbsbeteiligung korrespondiert. So zeigte Diehl (2002), dass türkische Migranten in Deutschland eher eine Einbürgerung planen, wenn ein höheres Bildungsniveau sowie eine kulturelle und identifikative Integration vorliegen. Weiter ist anzunehmen, dass höhere Religiosität mit einer geringeren Erwerbsbeteiligung korrespondiert, da stärker religiöse Personen eine geschlechterspezifische Arbeitsteilung mit der Frau im Haushalt präferieren (van Tubergen 2006; Diehl et al. 2009; Maliepard et al. 2010; Höhne/Koopmans 2010). Nach der Familieninvestitionshypothese (Dulepp 1998) ist davon auszugehen, dass die Erwerbsbeteiligung insbesondere unter verheirateten Frauen gering ist, insbesondere unter den Müttern (Brewster/Rindfuss 2000).

\section{Daten, Variablen und Methode}

Datengrundlage für die Studie ist der Generations and Gender Survey (GGS). Der GGS ist eine international vergleichende Bevölkerungsumfrage zur Untersuchung von Familien- und Geschlechterbeziehungen in Industrieländern, die international von der Economic Comission for Europe der Vereinten Nationen in Genf bzw. für Deutschland vom Bundesinstitut für Bevölkerungsforschung, Wiesbaden, koordiniert wird (Ette et al. 2007). In Deutschland fand die erste Datenerhebung der Hauptbefragung 2005 mit 10.017 Interviews statt; 2006 wurde die erste Zusatzerhebung von 4045 in Deutschland lebenden türkischen Staatsbürgern durchgeführt (vgl. Ette et al. 2007). 
Das für diese Analyse genutzte Sample beschränkt sich auf Frauen im erwerbsfähigen Alter von 18 bis 65 Jahren (das gesetzliche Renteneintrittsalter lag in den Jahren 2005 bis 2006 bei 65 Jahren). Personen, die sich zum Zeitpunkt der Befragung in Ausbildung (Lehre/Studium) befanden, wurden ausgeschlossen wie auch Rentnerinnen, permanent kranke Personen und diejenigen Fälle ohne Angabe zur Erwerbstätigkeit.

Als Personen mit türkischem Migrationshintergrund wurden sowohl aus der Zusatzbefragung türkischer Staatsbürger 2006 als auch aus der ersten Hauptbefragung 2005 alle Fälle klassifiziert, bei denen das Geburtsland die Türkei ist oder wenigstens ein Elternteil in der Türkei geboren wurde. Das Sample umfasst 1643 Frauen. Zur Unterscheidung der Migrantengeneration wurde das Zuzugsalter 16 herangezogen ${ }^{1}$ : Frauen, die einschließlich bis zum Alter 15 aus der Türkei immigriert sind oder in Deutschland als Kind von mindestens einem Elternteil aus der Türkei geboren wurden, gehören der zweiten Generation an; dies sind 740 Frauen (45 Prozent). 903 (55 Prozent) Frauen mit höherem Alter bei der Migration gehören der ersten Migrantengeneration an.

Das Alter der Befragten wird aufgrund des U-förmigen Zusammenhangs mit der abhängigen Variablen in drei Kategorien erfasst. Etwa 11 Prozent der Türkinnen waren zum Zeitpunkt der Befragung 18 bis 24 Jahre alt, zwei Drittel 25 bis 44 Jahre alt und 23 Prozent 45 bis 65 Jahre alt, wobei die Altersstruktur der zweiten Generation jünger ist als die der ersten.

\section{Variablen}

Die abhängige Variable ist die Erwerbsbeteiligung. Als Erwerbspersonen werden Frauen klassifiziert, die zum Zeitpunkt der Befragung abhängig oder selbstständig beschäftigt, mithelfende Familienangehörige oder in Mutterschutz/Erziehungszeit waren oder als Erwerbsstatus ,arbeitslos“ angegeben haben. Als Nichterwerbspersonen/Hausfrauen werden Frauen definiert, die auf die Frage nach dem Erwerbsstatus mit „Hausfrau“ geantwortet haben. Rund 53 Prozent im Sample sind Hausfrauen und etwa 47 Prozent sind Erwerbspersonen. Der Anteil der Hausfrauen unterscheidet sich signifikant zwischen den Migrantengenerationen $(p<0,001)$ : Während in der ersten Generation 66 Prozent Hausfrauen sind, beträgt dieser Anteil in der zweiten Generation 37 Prozent (siehe Tabelle 1; zum Vergleich: 17 Prozent der Nichtmigrantinnen sind nicht erwerbsbeteiligt, GGS 2005).

Für alle unabhängigen Variablen wurden bivariate Analysen durchgeführt, um Unterschiede zwischen der ersten und zweiten Migrantengeneration zu testen. Die Migrantengenerationen unterscheiden sich in den aufgeführten Variablen signifikant voneinander (siehe Tabelle 1). Als Indikatoren für die intergenerationale Transmission wurden zwei Variablen der Mutter generiert: Mehr als die Hälfte der Frauen hat Mütter (54 Prozent), die keinen Bildungsabschluss haben. In etwa 22 Prozent der Fälle haben die Mütter eine Primärbildung und in etwa drei Prozent eine Sekundarausbildung (etwa 21 Prozent sonstige oder fehlende Angaben). In drei Viertel der Fälle gaben die Befragten an, dass ihre Mutter Hausfrau war, als die Befragten 15 Jahre alt waren (83 Prozent in der ersten und 65 Prozent in der zweiten Generation).

1 Die Definition der zweiten Migrantengeneration erfolgt in der Literatur unterschiedlich, je nach Fragestellung, wobei für Untersuchungen zu Bildung und Arbeitsmarkt meist Alter angenommen werden, die mit Übergängen ins oder im Bildungssystem verbunden sind (u.a. Rumbaut 2004; Söhn 2011). 
Als ein $\mathrm{Ma}$ für die Generationenbeziehungen bzw. intergenerationale Unterstützung dient die Angabe, ob mindestens ein Elternteil der Befragten oder ihres Partners im selben Haushalt wohnt (Deindl/Isengard 2011; Zimmermann 2012). Dies trifft auf sieben Prozent der Stichprobe zu. Wenn ein Elternteil im Haushalt wohnt, ist die Erwerbsbeteiligung höher $(p=0,006)$. Zudem werden im GGS vier Einstellungsvariablen zur Pflege der Eltern durch ihre Kinder erfragt, deren Antworten in einer Likert-Skala erfasst werden. Der Anteil derjenigen, die diesen Aussagen zustimmen, ist mit etwa 36 Prozent am niedrigsten bei der Frage nach Geschlechterunterschieden in der Pflege der Eltern (Item: „Eher Töchter als Söhne sollen sich um Probleme der Eltern kümmern" ") ${ }^{2}$. Laut Zimmermann (2012) ist die Teilung in den Verantwortlichkeiten nach Geschlecht sowohl in der islamischen Religion als auch in der türkischen Kultur verankert. Wenngleich diese Variable in der bivariaten Statistik nicht in einem signifikanten Zusammenhang mit der Erwerbsbeteiligung steht, so unterscheiden sich doch die Migrantengenerationen in ihrer Einstellung zur geschlechterspezifischen Pflege auf dem 0,1\%-Niveau. Zudem wurde ein Indikator gebildet, der aufsummiert, ob die Befragten den anderen drei Items zur Elternpflege Zustimmung entgegen bringen (51 Prozent stimmten allen drei Ansichten zu; 41 Prozent stimmten ein- oder zweimal oder gar nicht zu; $\mathrm{p}=0,008$ ). Die Zustimmung zur Pflege nimmt in der zweiten Generation ab.

Insbesondere für die Erwerbstätigkeit von Frauen sind die Anzahl der Kinder sowie das Alter des jüngsten Kindes von Bedeutung (Brewster/Rindfuss 2000, Uunk et al. 2005; Rendall et al. 2010). Etwa ein Viertel der Frauen lebt ohne Kinder im Haushalt, 21 Prozent haben ein Kind, etwa 31 Prozent haben zwei bzw. 24 Prozent haben drei und mehr Kinder. Die Erwerbsbeteiligung sinkt mit höherer Anzahl der Kinder $(p<0,001)$.

Um die Unterstützung von Kindern durch ihre Eltern zu berücksichtigen, wurde eine Dummyvariable generiert für diejenigen, die in der Betreuung ihrer Kinder Unterstützung durch ihre Eltern erhalten (Attias-Donfut 2000). Dies sind etwa 10 Prozent des Samples. Da Anzahl und Alter der Kinder sowie Kinderbetreuung hoch korreliert sind, wird nur der Dummy für Betreuung durch die Großeltern berücksichtigt, nicht aber das Alter des jüngsten Kindes.

Die Variable Schulabschluss erfasst die Kategorien kein Schulabschluss (26 Prozent), Primärbildung (Hauptschule; 43 Prozent), untere Sekundarbildung (Realschule; 18 Prozent) und obere Sekundarbildung (Fach-/Hochschulreife; 8 Prozent); etwa sechs Prozent in diesem Sample haben einen anderen Abschluss oder fehlende Angaben. In der bivariaten Statistik ist die Erwerbsbeteiligung höher, wenn Frauen eine höhere Schulbildung haben $(p<0,001$, Becker 1991), wobei der Anteil derjenigen ohne Schulabschluss in der zweiten Generation deutlich niedriger ist als der in der ersten Migrantengeneration $(\mathrm{p}<0,001)$.

Eine zweite Bildungsvariable beinhaltet die berufliche Qualifikation bzw. Tertiärbildung. Knapp 67 Prozent der Stichprobe haben keine berufliche Qualifikation erworben. 32 Prozent haben eine Berufsausbildung abgeschlossen, ein Studium oder eine Meisterausbildung absolviert (aufgrund der mit etwa drei Prozent zu geringen Fallzahl für Studium und Meisterausbildung wurden die drei Kategorien zusammengefasst). In der bivariaten Statistik ist die Erwerbsbeteiligung höher, wenn Frauen eine berufliche Qualifikation

2 Die anderen drei Items sind: „Kinder sollen Verantwortung für Eltern übernehmen, wenn diese Hilfe brauchen“ (91 Prozent Zustimmung); „Kinder sollen Arbeitsleben umorganisieren, wenn Eltern Hilfe brauchen“ (55 Prozent Zustimmung); „Kinder sollen Eltern bei Problemen im Alter zu sich nehmen“" (81 Prozent Zustimmung). 
haben $(p<0,001)$, und der Anteil der Frauen ohne berufliche Ausbildung ist in der zweiten Generation geringer als der in der ersten $(p<0,001)$.

Etwa acht Prozent der Frauen mit türkischem Migrationshintergrund haben die deutsche Staatsbürgerschaft; und ihre Erwerbsbeteiligung ist höher $(\mathrm{p}<0,001)$.

95 Prozent des Samples geben an, einer muslimischen Religionsgemeinschaft anzugehören. Religiosität wurde im GGS anhand von fünf Fragen erhoben, die auch in der $\mathrm{Zu}$ satzbefragung türkischer Staatsbürger an die Fragen aus der Hauptbefragung angelehnt waren. Dies sind die Häufigkeit der Teilnahme an Gottesdiensten/religiösen Veranstaltungen, die Bedeutung religiöser Rituale - Taufe, Heirat, Beerdigung - sowie die Wichtigkeit religiöser Erziehungsziele. Da diese Fragen eher auf einen christlichen Kontext zugeschnitten sind, resultieren die zum Teil relativ hohen Antwortausfälle bei Muslimen vermutlich auch aus einem Unverständnis der Fragen. In dieser Analyse wird der Besuch religiöser Veranstaltungen als Indikator für Religiosität herangezogen. Etwa 33 Prozent der Stichprobe gaben an, dass sie mindestens monatlich eine religiöse Veranstaltung besuchen; etwa 67 Prozent nehmen entsprechend seltener oder nie an einer Veranstaltung teil, wobei sich in der zweiten Generation ein Rückgang der Religiosität findet. Es ist anzunehmen, dass höhere Religiosität mit einer geringeren Erwerbsbeteiligung einher geht (van Tubergen 2006; Maliepard et al. 2010), was sich in der bivariaten Statistik findet $(\mathrm{p}<0,001)$. Die Teilnahmehäufigkeit an religiösen Veranstaltungen ist zur Messung von Religiosität, insbesondere unter Muslimen, durchaus umstritten, da der Besuch des (Freitags-)Gebetes für Männer, nicht aber für Frauen im Islam die Norm ist (u.a. van Tubergen 2006). Dies ist m.E. vor allem für einen Vergleich von Religiosität zwischen Muslimen und Christen bzw. zwischen Männern und Frauen problematisch. Da in der vorliegenden Analyse jedoch nur Frauen mit türkischem Migrationshintergrund betrachtet werden, sollte diese Variable zumindest einen Hinweis auf den Einfluss eines religiös organisierten Umfeldes auf die Erwerbstätigkeit geben. Alternativ wurden auch die anderen Variablen zur Religiosität ausgewertet. Die Frage nach der Taufe wurde vom Großteil der Befragten nicht beantwortet, was vermutlich auf ein Verständnisproblem zurückzuführen ist, da es im Islam kein echtes Äquivalent dazu gibt. Religiöse Heirat und Beerdigung erscheinen inhaltlich als eher weniger mit der Erwerbsbeteiligung von Frauen verknüpft. Etwa 28 Prozent des Samples nannten Religion als eines der drei wichtigsten Erziehungsziele. Vorteil der Verwendung von Erziehungsziel gegenüber Veranstaltung wäre, dass dieser Indikator weniger in der symbolischen oder ritualistischen Dimension von Religiosität angesiedelt (Glock 2006; Dieh1/Koenig 2009), sondern mehr in der Alltagswelt verankert wäre. Glock (2006) bezeichnet dies als „consequential dimension“, wobei sich Religion und Religiosität in Einstellungen, Normen und Verhalten übersetzen. Die Variablen Erziehungsziele und Elternschaft bzw. Kinderzahl korrelieren jedoch stark miteinander, sodass auch diese Variable als weniger geeignet erscheint.

Zum Familienstand liegt im GGS keine direkt verwendbare Variable vor. Befragte gaben an, ob sie mit dem in ihrem Haushalt lebenden Partner verheiratet sind beziehungsweise eine eingetragene Lebenspartnerschaft führen oder ob sie außerhalb des eigenen Haushalts eine intime Partnerschaft führen. Aus diesen Angaben wurde die Variable Partnerschaftsstatus generiert. Etwa vier Fünftel der Frauen mit türkischem Migrationshintergrund leben mit ihrem Partner in einem Haushalt. Etwa drei Prozent davon sind nichteheliche Lebensgemeinschaften (NEL). Daher werden Ehen und NEL als Lebensgemeinschaften zusammengefasst. Etwa 17 Prozent berichteten keine Partnerschaft (diese Gruppe beinhaltet Ledige wie auch Verwitwete und Geschiedene, die fünf Prozent des Samp- 
les ausmachen), und drei Prozent gaben an, einen Partner (nicht im Haushalt) zu haben. Die Erwerbsbeteiligung ist unter den verheirateten Frauen gering, insbesondere unter Müttern ( $\mathrm{p}<0,001$, Brewster/Rindfuss 2000).

Tabelle 1: Überblick über die Stichprobe, nach Migrantengeneration

\begin{tabular}{|c|c|c|c|c|c|c|}
\hline \multirow[b]{2}{*}{ Variablen } & \multicolumn{2}{|c|}{ Gesamt } & \multicolumn{2}{|c|}{ 1. Generation } & \multicolumn{2}{|c|}{ 2. Generation } \\
\hline & $\mathbf{N}$ & $\%$ & $\mathbf{N}$ & $\%$ & $\mathbf{N}$ & $\%$ \\
\hline \multicolumn{7}{|l|}{ Erwerbsbeteiligung ${ }^{* * *}$} \\
\hline Hausfrau & 866 & 52,7 & 592 & 65,6 & 274 & 37,0 \\
\hline Erwerbsperson & 777 & 47,3 & 311 & 34,4 & 466 & 63,0 \\
\hline \multicolumn{7}{|l|}{ Sozio-demografische Merkmale der Frau } \\
\hline \multicolumn{7}{|l|}{ Alter ${ }^{* * *}$} \\
\hline 18 bis 24 Jahre & 177 & 10,5 & 57 & 6,3 & 120 & 16,2 \\
\hline 25 bis 44 Jahre & 1089 & 66,0 & 523 & 57,9 & 566 & 76,5 \\
\hline 45 bis 65 Jahre & 377 & 23,5 & 323 & 35,8 & 54 & 7,3 \\
\hline \multicolumn{7}{|l|}{ Schulbildung ${ }^{\star \star *}$} \\
\hline Kein Schulabschluss & 431 & 26,2 & 341 & 37,8 & 90 & 12,2 \\
\hline Hauptschule & 700 & 42,6 & 342 & 37,9 & 358 & 48,4 \\
\hline Realschule & 293 & 17,8 & 88 & 9,7 & 205 & 27,7 \\
\hline Fach-/Hochschulreife & 126 & 7,7 & 55 & 6,1 & 71 & 9,6 \\
\hline Andere/keine Angaben & 93 & 5,7 & 77 & 8,5 & 16 & 2,2 \\
\hline \multicolumn{7}{|l|}{ Berufliche Qualifikation ${ }^{\star \star *}$} \\
\hline Keine & 1099 & 66,9 & 717 & 79,4 & 382 & 51,6 \\
\hline Lehre/Meister/Hochschulabschluss & 529 & 32,2 & 178 & 19,7 & 351 & 47,4 \\
\hline Andere/keine Angaben & 15 & 0,9 & 8 & 0,9 & 7 & 0,9 \\
\hline \multicolumn{7}{|l|}{ Staatsangehörigkeit ${ }^{* * *}$} \\
\hline Deutsch & 125 & 7,6 & 17 & 1,9 & 108 & 14,6 \\
\hline Türkisch & 1518 & 92,4 & 886 & 98,1 & 632 & 85,4 \\
\hline \multicolumn{7}{|l|}{ Besuch von religiösen Veranstaltungen $n^{\star \star *}$} \\
\hline Einmal pro Monat und öfter & 545 & 33,2 & 355 & 39,3 & 190 & 25,7 \\
\hline Weniger als monatlich bis nie/keine Angabe & 1098 & 66,8 & 548 & 60,7 & 550 & 74,3 \\
\hline \multicolumn{7}{|l|}{ Partnerschaftsstatus ${ }^{* * *}$} \\
\hline Lebensgemeinschaft (verheiratet/unverheiratet) & 1295 & 78,8 & 770 & 85,3 & 525 & 71,0 \\
\hline Partnerschaft & 43 & 2,6 & 7 & 0,8 & 36 & 4,9 \\
\hline Keine Partnerschaft (ledig/verwitwet/geschieden) & 275 & 16,7 & 117 & 13,0 & 158 & 21,4 \\
\hline Keine Angabe & 30 & 1,8 & 9 & 1,0 & 21 & 2,8 \\
\hline \multicolumn{7}{|l|}{ Anzahl der Kinder im Haushalt*** } \\
\hline Keine Kinder & 402 & 24,5 & 185 & 20,5 & 217 & 29,3 \\
\hline 1 Kind & 344 & 20,9 & 199 & 22,0 & 145 & 19,6 \\
\hline 2 Kinder & 503 & 30,6 & 281 & 31,1 & 222 & 30,0 \\
\hline 3 Kinder und mehr & 394 & 24,0 & 238 & 26,4 & 156 & 21,1 \\
\hline \multicolumn{7}{|l|}{ Intergenerationale Transmission } \\
\hline \multicolumn{7}{|l|}{ Bildung der Mutter*** } \\
\hline Kein Schulabschluss & 886 & 53,9 & 511 & 56,6 & 375 & 50,7 \\
\hline Hauptschule & 359 & 21,9 & 144 & 15,9 & 215 & 29,1 \\
\hline Realschule/ Fach-/Hochschulabschluss & 48 & 2,9 & 20 & 2,2 & 28 & 3,8 \\
\hline Andere/keine Angabe & 350 & 21,3 & 228 & 25,2 & 122 & 16,5 \\
\hline
\end{tabular}




\begin{tabular}{|c|c|c|c|c|c|c|}
\hline \multirow[b]{2}{*}{ Variablen } & \multicolumn{2}{|c|}{ Gesamt } & \multicolumn{2}{|c|}{ 1. Generation } & \multicolumn{2}{|c|}{ 2. Generation } \\
\hline & $\mathbf{N}$ & $\%$ & $\mathbf{N}$ & $\%$ & $\mathbf{N}$ & $\%$ \\
\hline \multicolumn{7}{|c|}{ Mutter war Hausfrau, als Befragte 15 Jahre alt war*** } \\
\hline $\mathrm{Ja}$ & 1227 & 74,7 & 749 & 82,9 & 478 & 64,6 \\
\hline Nein/keine Angabe & 416 & 25,3 & 154 & 17,1 & 262 & 35,4 \\
\hline \multicolumn{7}{|l|}{ Generationenbeziehungen } \\
\hline \multicolumn{7}{|l|}{ Mindestens 1 Elternteil im Haushalt***} \\
\hline $\mathrm{Ja}$ & 111 & 6,8 & 27 & 3,0 & 84 & 11,4 \\
\hline Nein & 1532 & 93,2 & 876 & 97,0 & 656 & 88,6 \\
\hline \multicolumn{7}{|c|}{ Töchter sollen sich um Probleme der Eltern kümmern *** } \\
\hline Stimme eher zu & 598 & 36,4 & 362 & 40,1 & 236 & 31,9 \\
\hline Stimme eher nicht zu & 699 & 42,5 & 334 & 37,0 & 365 & 49,3 \\
\hline Weiß nicht/keine Angabe & 346 & 21,1 & 207 & 22,9 & 139 & 18,8 \\
\hline \multicolumn{7}{|l|}{ Zustimmung zu Elternpflege durch Kinder ${ }^{* * *}$} \\
\hline Nie, 1 - oder 2 Items & 836 & 50,9 & 421 & 46,6 & 415 & 56,1 \\
\hline 3 Items & 807 & 49,1 & 482 & 53,4 & 325 & 43,9 \\
\hline \multicolumn{7}{|c|}{ Unterstützung bei Kinderbetreuung durch Großeltern ${ }^{(1) * * *}$} \\
\hline Keine Unterstützung durch Großeltern & 1117 & 90,0 & 678 & 94,4 & 439 & 83,9 \\
\hline Unterstützung durch Eltern/Schwiegereltern & 124 & 10,0 & 40 & 5,6 & 84 & 16,1 \\
\hline$N$ & 1643 & 100,0 & 903 & 55,0 & 740 & 45,0 \\
\hline
\end{tabular}

1) Nur Anteile der Frauen, die Kinder im Haushalt haben.

Anmerkung: *** $\mathrm{p}<=0,001$.

Quelle: GGS-D 2005/06 (eigene Berechnungen).

\section{Methode und Modellstrategie}

Die abhängige Variable der logistischen Regressionsanalyse ist die Erwerbsbeteiligung gegenüber der Hausfrauentätigkeit. Die Ergebnisse werden als Odds Ratio (OR, „Effektkoeffizienten") dargestellt (Best/Wolf 2010). Die Modellschätzung erfolgt schrittweise: In Modell 1 wird der Bruttoeffekt für die Generationenvariable geschätzt; dabei wird nur als Kontrollvariable das Alter der Frau eingefügt, um die unterschiedlichen Altersstrukturen der beiden Gruppen zu berücksichtigen. Die Modelle 2 und 3 fügen die Variablen zu den Generationenbeziehungen und zur intergenerationalen Transmission hinzu. Modell 4 ergänzt die sozio-demografischen Merkmale der Frau sowie Haushaltsvariablen in Modell 5. Im Anschluss erfolgt eine zusammenfassende Darstellung alternativer Modellspezifikationen.

\section{Resultate}

Tabelle 2 stellt die Ergebnisse der logistischen Regressionsanalyse zusammen. Modell 1 zeigt, dass die Erwerbsbeteiligung unter Frauen der zweiten Migrantengeneration mehr als dreimal so hoch ist wie in der ersten. In den Altersklassen bis 24 und über 45 Jahren ist die Erwerbsbeteiligung signifikant erhöht, verglichen mit Frauen im Alter von 25 bis 44 Jahren. 
Tabelle 2: Einflussfaktoren der Erwerbsbeteiligung (odds ratios)

\begin{tabular}{|c|c|c|c|c|c|}
\hline Variablen & Modell 1 & Modell 2 & Modell 3 & Modell 4 & Modell 5 \\
\hline \multicolumn{6}{|l|}{ Migrantengeneration } \\
\hline Erste Generation & 1 & 1 & 1 & 1 & 1 \\
\hline Zweite Generation & $3,57^{\star \star \star}$ & $3,48^{* * *}$ & $2,96^{\star * *}$ & $2,24^{\star * *}$ & $2,03^{\star * *}$ \\
\hline \multicolumn{6}{|l|}{ Alter } \\
\hline 18 bis 24 Jahre & $1,40^{\circ}$ & $1,43^{*}$ & 1,07 & 1,01 & 0,66 \\
\hline 25 bis 44 Jahre & 1 & 1 & 1 & 1 & 1 \\
\hline 45 bis 65 Jahre & $1,53^{* *}$ & $1,57^{* * *}$ & $1,59^{* * *}$ & $1,92^{\star * *}$ & 1,28 \\
\hline \multicolumn{6}{|l|}{ Töchter sollen sich um Eltern kümmern*** } \\
\hline Stimme eher zu & & 1 & 1 & 1 & 1 \\
\hline Stimme eher nicht zu & & $1,36^{*}$ & $1,35^{*}$ & 1,19 & 1,30 \\
\hline Weiß nicht/keine Angabe & & 1,25 & $1,27^{\circ}$ & 1,23 & $1,37^{*}$ \\
\hline \multicolumn{6}{|l|}{ Zustimmung zu Elternpflege durch Kinder ${ }^{* * *}$} \\
\hline Nie, 1- oder 2 Items & & 1,10 & 1,07 & 0,98 & 0,97 \\
\hline 3 Items & & 1 & 1 & 1 & 1 \\
\hline \multicolumn{6}{|l|}{ Mindestens 1 Elternteil im Haushalt*** } \\
\hline $\mathrm{Ja}$ & & & $4,08^{\star * *}$ & $4,41^{* * *}$ & $1,89^{*}$ \\
\hline Nein & & & 1 & 1 & 1 \\
\hline \multicolumn{6}{|l|}{ Bildung der Mutter ${ }^{\star \star *}$} \\
\hline Kein Schulabschluss & & & 0,81 & $1,11^{\circ}$ & 1,06 \\
\hline Hauptschule & & & 1 & 1 & 1 \\
\hline Realschule/ Fach-/Hochschulabschluss & & & 0,74 & 0,57 & 0,51 \\
\hline Andere/keine Angabe & & & $0,76^{\circ}$ & 1,04 & 0,98 \\
\hline \multicolumn{6}{|c|}{ Mutter war Hausfrau, als Befragte 15 Jahre alt war*** } \\
\hline $\mathrm{Ja}$ & & & 1 & 1 & 1 \\
\hline Nein/keine Angabe & & & $1,76^{\star \star *}$ & $1,65^{\star * \star}$ & $1,55^{\star *}$ \\
\hline \multicolumn{6}{|l|}{ Schulbildung } \\
\hline Kein Schulabschluss & & & & $0,77^{\circ}$ & $0,73^{*}$ \\
\hline Hauptschule & & & & 1 & 1 \\
\hline Realschule & & & & 1,26 & 1,15 \\
\hline Fach-/Hochschulreife & & & & 1,48 & 1,33 \\
\hline Andere/keine Angabe & & & & 0,81 & 0,75 \\
\hline \multicolumn{6}{|l|}{ Berufliche Qualifikation } \\
\hline Keine & & & & 1 & 1 \\
\hline Lehre/Meister/Hochschulabschluss & & & & $2,37^{\star * *}$ & $2,10^{\star * *}$ \\
\hline Andere/keine Angabe & & & & 1,36 & 1,49 \\
\hline \multicolumn{6}{|l|}{ Staatsangehörigkeit } \\
\hline Deutsch & & & & $1,50^{\circ}$ & $1,58^{\circ}$ \\
\hline Türkisch & & & & 1 & 1 \\
\hline \multicolumn{6}{|l|}{ Besuch von religiösen Veranstaltungen } \\
\hline Einmal pro Monat und öfter & & & & 1 & 1 \\
\hline Weniger als monatlich bis nie/ keine Ang & & & & $1,63^{* * *}$ & $1,51^{* *}$ \\
\hline \multicolumn{6}{|l|}{ Partnerschaftsstatus ${ }^{* * *}$} \\
\hline Lebensgemeinschaft (verheiratet/unverhe & atet) & & & & 1 \\
\hline Partnerschaft & & & & & $4,42^{* *}$ \\
\hline Keine Partnerschaft (ledig/verwitwet/gesc & eden) & & & & $3,49^{* * *}$ \\
\hline Keine Angabe & & & & & 2,56 \\
\hline
\end{tabular}




\begin{tabular}{|c|c|c|c|c|c|}
\hline Variablen & Modell 1 & Modell 2 & Modell 3 & Modell 4 & Modell 5 \\
\hline \multicolumn{6}{|l|}{ Anzahl der Kinder im Haushalt*** } \\
\hline Keine Kinder & & & & & 1 \\
\hline 1 Kind & & & & & $0,59^{* *}$ \\
\hline 2 Kinder & & & & & $0,40^{* * *}$ \\
\hline 3 Kinder und mehr & & & & & $0,35^{* * *}$ \\
\hline \multicolumn{6}{|c|}{ Unterstützung bei Kinderbetreuung durch Großeltern *** } \\
\hline Keine Unterstützung durch Großeltern & & & & & 1 \\
\hline Unterstützung durch Eltern/Schwiegerelterr & & & & & 1,08 \\
\hline$L L$ & $-1062,88$ & $-1058,31$ & $-1029,07$ & $-978,797$ & $-916,31$ \\
\hline$R^{2}$ & 0,06 & 0,07 & 0,09 & 0,14 & 0,19 \\
\hline
\end{tabular}

Anmerkung: *** $\mathrm{p}<=0,001, * * \mathrm{p}<=0,01,{ }^{*} \mathrm{p}<=0,05,{ }^{\circ} \mathrm{p}<=0,1$.

Quelle: GGS-D 2005/06 (eigene Berechnungen); N=1643.

Modell 2 fügt die Einstellungen zur Unterstützung der Eltern hinzu. Während die Anzahl der Zustimmungen zur Elternpflege keinen Einfluss zu haben scheint, ist ein signifikanter Unterschied in der Genderrollenvariablen zu erkennen, die sich auch leicht auf den Generationenunterschied auswirkt: Die Erwerbsbeteiligung ist unter jenen Frauen etwa ein Drittel höher, die nicht die Meinung teilen, dass Kinder durch ihre Töchter und weniger durch ihre Söhne unterstützt werden sollten.

Modell 3 berücksichtigt die Indikatoren für die intergenerationale Transmission (Bildung der Mutter, Erwerbsstatus der Mutter) sowie die Frage nach den im Haushalt lebenden Eltern: Die Schulbildung der Mutter wirkt sich insofern aus, als dass mit höherer Bildung der Mutter die Erwerbsbeteiligung ihrer Tochter steigt. Dieser Effekt ist allerdings nicht signifikant, da er zum einen von der beruflichen Bildung der Tochter und zum anderen von einem der wichtigsten Prädiktoren aufgegriffen wird: War die Mutter erwerbstätig, ist die eigene Erwerbsbeteiligung signifikant um etwa 76 Prozent erhöht im Vergleich zu Frauen, deren Mütter Hausfrauen waren. Ein im Haushalt wohnender (Schwieger-)Elternteil erhöht die Erwerbsbeteiligung um etwa das Vierfache. Durch die Hinzunahme dieser drei Variablen verringert sich der Generationeneffekt nur leicht; die Unterschiede in der Einstellung der Genderrollen in der Elternpflege bleiben ebenfalls signifikant.

In Modell 4 verringert sich der Generationeneffekt zwar durch die Hinzunahme der Humankapitalfaktoren; die Erwerbsbeteiligung bleibt unter Migrantennachkommen dennoch stark signifikant erhöht. Die Kontrollvariablen selbst weisen die angenommenen $\mathrm{Zu}-$ sammenhänge auf: Die Erwerbsbeteiligung steigt mit zunehmender Schulbildung. Diese Variable korreliert allerdings mit der beruflichen Qualifikation - sobald zusätzlich die berufliche Ausbildung berücksichtigt wird, wird der Schulbildungseffekt insignifikant. Jedoch ist dann zu sehen, dass Frauen mit beruflicher Qualifikation eine doppelt so hohe Erwerbsbeteiligung haben wie jene ohne Lehrausbildung. Ebenso geht ein Vorhandensein der deutschen Staatsbürgerschaft mit einer signifikant höheren Erwerbsbeteiligung einher. Schaut man sich den Indikator für Religiosität an, haben diejenigen, die mindestens monatlich an einer religiösen Veranstaltung teilnehmen, eine signifikant niedrigere Erwerbsbeteiligung als jene, die seltener bis nie religiöse Veranstaltungen besuchen. Unter Berücksichtigung der Bildung der Frau nivelliert sich der Einfluss der Genderrollenvariablen.

In Modell 5 werden Indikatoren für die Familiensituation hinzugefügt. Erwartungsgemäß sind ledige Frauen am seltensten Hausfrauen bzw. ist die Erwerbsbeteiligung in Lebensgemeinschaften am geringsten (Ergebnisse nicht dargestellt). Dieser Effekt weicht 
jedoch, wenn die Anzahl der Kinder im Haushalt berücksichtigt wird: Je mehr Kinder eine Frau hat, desto niedriger ist ihre Erwerbsbeteiligung gegenüber Kinderlosen. Eine Hilfe bei der Kinderbetreuung durch Großeltern wirkt sich nicht signifikant auf die Erwerbsbeteiligung aus, wenn man gleichzeitig im Modell kontrolliert, ob ein Elternteil der Frau oder ihres Partners im selben Haushalt wohnt, sodass man davon ausgehen kann, dass in Drei-Generationen-Haushalten die Unterstützung der älteren Generation an die Jüngeren dazu beiträgt, die Erwerbsbeteiligung zu fördern. Unter Kontrolle dieser Einflussfaktoren verringert sich der Generationenunterschied leicht, bleibt jedoch signifikant erhöht; die Erwerbsbeteiligung ist unter Frauen der zweiten etwa doppelt so hoch wie in der ersten Migrantengeneration. Modell 5 hat mit einem $\mathrm{R}^{2}$ von etwa 0,20 die höchste Erklärungskraft dieser Modelle.

\section{Alternative Modellspezifikationen}

Alternativ wurde die Regressionsanalyse mit anderen erklärenden Variablen durchgeführt. In Modell 1 wurde die erste Migrantengeneration nach ihrer Aufenthaltsdauer unterschieden. Hier war mit zunehmendem Aufenthalt eine erhöhte Erwerbsbeteiligung festzustellen.

In Modell 2 wurden anstelle der Gender-Präferenzen in der Elternpflege die anderen drei Variablen zur Einstellung zur Elternpflege einzeln getestet. Keine von ihnen zeigte einen signifikanten Zusammenhang mit der Erwerbsbeteiligung.

In Modell 4 wurde Religiosität alternativ zur Teilnahme an religiösen Veranstaltungen durch die Frage nach den Erziehungszielen als Indikator für Religiosität benutzt. Hier unterscheiden sich die Migrantengenerationen nicht signifikant voneinander. In diesem Sample besteht zwischen der Häufigkeit der Teilnahme an religiösen Veranstaltungen und Religion als Erziehungsziel ein hochsignifikanter Zusammenhang $(\mathrm{p}<0,001)$, sodass dieser Indikator keine anderen Parameterschätzer (obgleich höchstsignifikant) erbringt als die Veranstaltungsvariable, weder im Effekt der Religiosität auf die Erwerbsbeteiligung noch für den Generationenvergleich.

$\mathrm{Zu}$ Modell 5: Da Partnerschaftsstatus und Kinderzahl stark miteinander in Zusammenhang stehen, wurden anstelle des alleinigen Vorhandenseins eines Partners (Modell 5) seine Bildung genutzt (Engelbrech 1987; van Tubergen 2008). Auch wurde der Erwerbsstatus des Partners berücksichtigt, da im Falle von Arbeitslosigkeit des Haushaltsvorstandes seine Partnerin sich möglicherweise eher selbst als arbeitssuchend denn als Hausfrau - im Einklang mit den Regelungen des Arbeitslosengeldes II - bezeichnet. Jedoch konnte für diese Partnervariablen kein signifikanter Einfluss auf die Erwerbsbeteiligung der Frau festgestellt werden. Zudem wurde das Erstheiratsalter der Frau benutzt. Hier zeigte sich, dass diejenigen, die vor dem Alter 25 geheiratet haben, eine signifikant niedrigere Erwerbsbeteiligung haben, als die Frauen, die später geheiratet haben oder aber ledig sind. Keine dieser Variablen erbrachte jedoch einen höheren Erklärungsgrad im Modell oder einen Unterschied für den Generationeneffekt. Dies gilt auch für die Familiengröße. Alternativ zur Anzahl der Kinder im Haushalt wurden die Gesamtzahl der Kinder und das Alter des jüngsten Kindes verwendet. Ähnlich der Kinderzahl im Haushalt sinkt die Erwerbsbeteiligung mit steigender Gesamtkinderzahl, während sie sich mit steigendem Alter des jüngsten Kindes erhöht. Modell 5 wurde zudem ohne den Partnerschaftsindikator 
gerechnet; dabei verringerte sich die Modellgüte jedoch signifikant; dies unterstreicht, dass die Partnerschaft zusätzlich zum Vorhandensein von Kindern einen Einfluss hat.

Schließlich wurde die abhängige Variable verändert. Da der Großteil der Frauen minderjährige Kinder im Haushalt hat und die Erwerbsbeteiligung vor allem bei jungen Müttern gering ist, wurden in einem alternativen Modell nur diejenigen als Hausfrauen definiert, die weder zum Zeitpunkt der Befragung noch jemals davor erwerbstätig waren. Von den türkischen Frauen insgesamt gaben 31 Prozent an, dass sie niemals erwerbstätig waren. Im Generationenvergleich waren dies 46 Prozent der ersten und 13 Prozent in der zweiten Migrantengeneration $(\mathrm{p}<0,001)$. In der Analyse wurden dieselben Schritte durchgeführt wie in Tabelle 2. Unter Kontrolle aller unabhängigen Variablen wie in Modell 5 beträgt der Generationenunterschied das 3,5-Fache mit höchster Signifikanz und einem $\mathrm{R} 2$ von 0,24 .

\section{Fazit}

Die vorliegende Studie hatte das Ziel die Inkorporation von Immigrantinnen auf dem Arbeitsmarkt in Deutschland zu untersuchen und dabei die Heterogenität der Migrantengruppe zu beachten. Dazu wurde mit Frauen aus der Türkei eine der größten Zuwanderergruppen im bedeutendsten Zielland Mittel- und Westeuropas herangezogen. Während in der bisherigen Forschung zur Arbeitsmarktintegration einerseits Männer und andererseits Arbeitsmarkt-Performance im Forschungsfokus standen, lag das Augenmerk dieser Analyse auf der Erwerbsbeteiligung von Frauen und der Rolle der Familie als förderndem oder hemmendem Faktor.

Im Sample des Generations and Gender Survey ist etwa jede zweite Frau mit türkischem Migrationshintergrund Hausfrau. Für die zweite Generation lässt sich jedoch eine drastisch gesteigerte Erwerbsbeteiligung feststellen. Während in der ersten Generation nur etwa 34 Prozent der Frauen Erwerbspersonen sind, beteiligen sich rund 63 Prozent in der zweiten Generation am Arbeitsmarkt. Die Entwicklung über die Generationen hinweg wird noch deutlicher, wenn man sich die Anteile derjenigen ansieht, die nie erwerbstätig waren: 46 Prozent der ersten und nur 13 Prozent in der zweiten Migrantengeneration sind permanent Hausfrauen.

Die Zunahme der Erwerbsbeteiligung mit steigender Aufenthaltsdauer innerhalb der ersten Generation und zwischen der ersten und der zweiten Generation spricht für die Arbeitsmarktassimilationshypothese der Generationen (H1) und steht im Einklang mit der Literatur. Eine große Rolle spielt hier zweifelsohne die höhere Bildungsbeteiligung in der zweiten Generation, auf die ein Teil des Generationenunterschiedes zurückzuführen ist.

Einen signifikanten Einfluss übt auch die intergenerationale Transmission aus (Hypothese 2): Die Erwerbsbeteiligung der Frau ist höher, wenn ihre Mutter bereits erwerbstätig war (Soremski 2008; Blau 2012), und dies ist in der zweiten Generation auch häufiger der Fall als in der ersten.

In Zusammenhang mit der Berufstätigkeit der Mutter und der Bildung stehen die Geschlechterrollen. Als Indikator wurde hier verwendet, ob bei der Pflege hilfebedürftiger Eltern eher Töchter als Söhne in der Pflicht sind, wie es in der Türkei die Norm ist. Die Ergebnisse der Analyse scheinen auch die Genderrollenhypothese (H4, Kawaguchi/Miyazaki 2009) zu unterstützen, wonach die Erwerbsbeteiligung unter denjenigen höher ist, 
die sich gegen geschlechtsspezifische Arbeitsteilung aussprechen. Jedoch zeigt sich bei Kontrolle der Bildungsvariablen der Frau, dass der Effekt der Geschlechterrollen für die Erwerbstätigkeit aus der Bildung resultiert: Mit höherer Bildung steigt die Ablehnung der geschlechterspezifischen Pflege, und bei Kontrolle der Bildung nivelliert sich der Gendereinstellungseffekt.

Schließlich ist auf die Hypothese zur intergenerationalen Unterstützung (H3) zurückzukommen. Es wurde mit Becker (1991) argumentiert, dass Normen der intergenerationalen Pflege, die speziell an die Frau herangetragen werden, die Erwerbsbeteiligung der Frau von vornherein senken, da Normen zur intergenerationalen Unterstützung und Traditionen der Frauenerwerbstätigkeit kulturell in den jeweiligen Ländern zusammenhängen. Bengtson/Roberts (1991) sprechen zum Beispiel von normativer Solidarität: Kinder und alternde Eltern sollten familiäre Verpflichtungen wahrnehmen, was zur Solidarität zwischen den Generationen beiträgt. Eine Erwerbstätigkeit würde somit potenziell für den Pflegefall Einschränkungen bedeuten. In der vorliegenden Analyse jedoch findet sich kein Beleg für diese These. Zwar geht die Zustimmung zur Pflegebereitschaft in der zweiten Generation zurück; dies wirkt sich aber nicht auf die Erwerbsbeteiligung aus.

Insgesamt ist zudem anzumerken, dass die Pflegebereitschaft in beiden Generationen recht hoch ist - dies widerspricht der Konfliktthese, wonach es durch den Migrationsprozess selbst oder durch strukturelle Entwicklungsprozesse zu einer Entfremdung der Generationen und zu einem Nachlassen der intergenerationalen Solidarität kommen würde. Zu einem ähnlichen Schluss kommt u.a. Baykara-Krumme et al. (2011) sowie Baykara-Krumme und Carnein/Baykara-Krumme (in diesem Heft). Vielmehr scheint es, dass die Entwicklung zu höherer Bildung unter Frauen mit türkischem Migrationshintergrund nicht die Generationenverhältnisse, aber die Einstellungen zu den tradierten Geschlechterrollen verändert, also weg von der Haushaltsspezialisierung mit der Frau in der Fürsorgerrolle, sondern hin zu eher egalitären Geschlechterrollen. Ähnlich wie höhere Bildung wirkt sich niedrigere Religiosität aus, die wiederum mit der Bildungsbeteiligung korreliert. Für die intergenerationalen Unterstützungsleistungen steht also nicht in Zweifel, $o b$ sich die Jüngeren um die Älteren sorgen wollen, sondern eher das Wer und Wie erscheinen gegenwärtig einem Wandel unterworfen.

Was die Rolle des Partners und der eigenen Kinder angeht, so findet auch diese Analyse - nicht überraschend -, dass die Erwerbsbeteiligung vor allem unter Müttern gering ist. Interessant ist, dass der Partnerschaftsstatus darüber hinaus einen eigenständigen Einfluss auf die Erwerbsbeteiligung hat. Geschlechterspezifisches Rollenverhalten setzt bei Frauen mit türkischem Migrationshintergrund mit einer (relativ frühen) Eheschließung ein, während ein Traditionalisierungsschub bei Deutschen eher erst mit dem ersten Kind zu beobachten ist (Wengler et al. 2008). Dies spricht dafür, dass Frauen mit türkischem Migrationshintergrund in Deutschland das traditionelle Familiengründungsmuster beibehalten, wonach Heirat und erstes Kind auch zeitlich eng verknüpft sind (Yavuz 2008; Milewski 2011).

$\mathrm{Zu}$ beachten ist, dass die höhere Erwerbsbeteiligung in der zweiten türkischen Generation nicht vollständig durch die Kontrollvariablen erklärt werden kann. So kann die intergenerationale Transmission - anders als dies beispielsweise für den (unterdurchschnittlichen) Bildungserfolg in der zweiten türkischen Migrantengeneration gilt, wonach intergenerationale Transmission als „Vererbung“ von Bildungsnachteilen die größte Erklärungskraft für die Stabilität ethnischer Ungleichheit im deutschen Schulsystem zuge- 
schrieben wird (Steinbach/Nauck 2004) - die Erwerbsbeteiligung von Migrantinnen nur wenig erklären. Dies deutet auf Anpassungsprozesse hin, die zusätzlich zu den hier geprüften strukturellen Entwicklungen ablaufen. Jedoch die Frage, wie viele der Frauen sich in die Hausfrauenrolle zurückgezogen haben, weil für sie die Barrieren auf dem Ausbildungs- und Arbeitsmarkt in Deutschland (zu) hoch sind, kann diese Studie nicht beantworten. Weitere Untersuchungen sollten daher die Rolle der sozialen Netzwerke bei der tatsächlichen Arbeitssuche und Beschäftigungssituation berücksichtigen.

\section{Dank}

Diese Studie wurde durch ein European Reintegration Grant innerhalb der Marie Curie Actions (FP7 People, PERG-GA-2009-249266 - MigFam) der Europäischen Kommission gefördert. Als wissenschaftliche Hilfskräfte wirkten Ellen von den Driesch und Kathrin Bahr mit. Mein Dank gilt auch den konstruktiven Vorschlägen der/des anonymen Gutachterin/Gutachters.

\section{Literatur}

Alba, R.D., Handl, J. \& Müller, W. (1994). Ethnische Ungleichheit im deutschen Bildungssystem. Kölner Zeitschrift für Soziologie und Sozialpsychologie, 46, S. 209-237.

Algan, Y., Dustmann, C., Glitz, A. \& Manning, A. (2010). The economic situation of first and secondgeneration immigrants in France, Germany and the United Kingdom. The Economic Journal, 120, S. 4-30.

Antecol, H. (2000). An examination of cross-country differences in the gender gap in labor force participation rates. Labour Economics, 7, 4, S. 409-426.

Attias-Donfut, C. (2000). Familialer Austausch und soziale Sicherung. In: Kohli, M. \& Szydlik, M. (Hrsg.), Generationen in Familie und Gesellschaft. Opladen: Leske + Budrich, S. 222-237.

Baykara-Krumme, H., Klaus, D. \& Steinbach, A. (2011). Generationenbeziehungen in Deutschland. Ein Vergleich der Beziehungsqualität in einheimischen deutschen Familien, Familien mit türkischem Migrationshintergrund und Aussiedlerfamilien. In: Brüderl, J., Castiglioni, L. \& Schumann, N. (Hrsg.), Partnerschaft, Fertilität und intergenerationale Beziehungen. Ergebnisse der ersten Welle des Beziehungs- und Familienpanels. Würzburg: Ergon, S. 259-286.

Becker, G. S. (1991). A treatise on the family. Cambridge, MA: Harvard University Press (2. Auflage).

von Below, S. \& Karakoyun, E. (2007). Sozialstruktur und Lebenslagen junger Muslime in Deutschland. In: von Wensierski, H.-J. \& Lübcke, C. (Hrsg.), Junge Muslime in Deutschland. Lebenslagen, Aufwachsprozesse und Jugendkulturen. Opladen \& Farmington Hills, MI: Verlag Barbara Budrich, S. 33-54.

Bengtson, V. L. \& Roberts, R. E. L. (1991). Intergenerational solidarity in aging families: An example of formal theory construction. Journal of Marriage and the Family, 53, 4, S. 856-870.

Best, H. \& Wolf, C. (2010). Logistische Regression. In: Best, H. \& Wolf, C. (Hrsg.), Handbuch der sozialwissenschaftlichen Datenanalyse. Wiesbaden: Springer VS, S. 827-854.

Biffl, G. (2002). Ausländische Arbeitskräfte auf dem österreichischen Arbeitsmarkt. Wien: Österreichisches Institut für Wirtschaftsforschung (WiFO Monatsberichte 8).

Blau, F. D., Kahn, L. M., Liu, A. Y.-H. \& Papps, K. L. (2012). The transmission of women's fertility, human capital, and work orientation across immigrant generation. Journal of Population Economics, doi: 10.1007/s00148-012-0424-x (online first).

Boos-Nünning, U. \& Karakaşoğlu, Y. (2005). Familialismus und Individualismus. Zur Bedeutung der Familie in der Erziehung von Mädchen mit Migrationshintergrund. In: Fuhrer, U. \& Uslucan, H.-H. 
(Hrsg.), Familie, Akkulturation und Erziehung. Migration zwischen Eigen- und Fremdkultur. Stuttgart: Kohlhammer, S. 126-149.

Bourdieu, P. (1983). Ökonomisches Kapital, kulturelles Kapital, soziales Kapital. In: Kreckel, R. (Hrsg.), Soziale Ungleichheiten. Göttingen: Schwarz, S. 183-198 (Sonderheft der Sozialen Welt, Band 2).

Brewster, K. L. \& Rindfuss, R. R. (2000). Fertility and women's employment in industrialized nations. Annual Review of Sociology, 26, S. 271-296.

Burkert, C., Hochfellner, D. \& Wurdack, A. (2012). Ältere Migrantinnen und Migranten am Arbeitsmarkt. In: Baykara-Krumme, H., Motel-Klingebiel, A. \& Schimany, P. (Hrsg.). Viele Welten des Alterns. Ältere Migranten im alternden Deutschland. Wiesbaden: Springer VS, S. 77-100.

Chiswick, B. R. (1978). The effect of Americanization on the earnings of foreign-born men. Journal of Political Economy, 86, S. 897-921.

Constant, A. F., Gataullina, L. \& Zimmermann, K. F. (2006). Gender, ethnic identity and work. Berlin: Deutsches Institut für Wirtschaftsforschung (DIW Discussion Paper 643).

Deindl, C. \& Isengard, B. (2011). Familiale Unterstützung und soziale Ungleichheit in Europa. In: Berger, P. A. (Hrsg.), Reproduktion von Ungleichheit durch Arbeit und Familie. Wiesbaden: Springer VS, S. 23-47.

Diehl, C. (2002). Wer wird Deutsche/r und warum? Bestimmungsfaktoren der Einbürgerung türkischund italienischstämmiger junger Erwachsener. Zeitschrift für Bevölkerungswissenschaft, 3, S. 285312.

Diehl, C. \& Koenig, M. (2009). Religiosität türkischer Migranten im Generationenverlauf: Ein Befund und einige Erklärungsversuche. Zeitschrift für Soziologie, 38, 4, S. 300-319.

Diehl, C., Koenig, M. \& Ruckdeschel, K. (2009). Religiosity and gender equality - Comparing natives and Muslim migrants in Germany. Ethnic and Racial Studies, 32, S. 278-301.

Duleep, H. O. (1998). The family investment model: A formalization and review of evidence across immigrant groups. Gender Issues, 16, S. 84-104.

El-Menouar, Y. \& Fritz, M. (2009). Sozioökonomische Entwicklung und Wertvorstellungen in elf Regionen der Türkei. Kölner Zeitschrift für Soziologie und Sozialpsychologie, 61, S. 535-561.

Engelbrech, G. (1987). Erwerbsverhalten und Berufsverlauf von Frauen: Ergebnisse neuerer Untersuchungen im Überblick. Nürnberg: Institut für Arbeitsmarkt und Berufsforschung (Mitteilungen aus der Arbeitsmarkt- und Berufsforschung 20), S. 2.

Ette, A., Hullen, G., Leven, I., \& Ruckdeschel, K. (2007). Generations and Gender Survey. In: Dokumentation der Befragung von türkischen Migranten in Deutschland. Wiesbaden: Bundesinstitut für Bevölkerungsforschung.

Fincke, G. (2009). Abgehängt, chancenlos, unwillig? Eine empirische Reorientierung von Integrationstheorien zu MigrantInnen der zweiten Generation in Deutschland. Wiesbaden: VS Verlag für Sozialwissenschaften.

Fleischmann, F. \& Dronkers, J. (2010). Unemployment among immigrants in European labor markets: An analysis of origin and destination effects. Work, Employment and Society, 24, 2, S. 337-354.

Glock, C. (2006 [1962]). On the study of religious commitment. Religious Education, 57, S4, S. 98-110.

de Graaf, N. D. \& Flap, H. D. (1988). With a little help from my friends: Social resources as an explanation of occupational status and income in West Germany, the Netherlands, and the United States. Social Forces, 67, S. 452-472.

Granato, N. (2003). Ethnische Ungleichheit auf dem deutschen Arbeitsmarkt. Opladen: Leske + Budrich (Schriftenreihe des Bundesinstituts für Bevölkerungsforschung 33).

Granovetter, M. (1973). The strength of weak ties. American Journal of Sociology, 78, S. 1360-1380.

Hancioğlu, A. \& Ergöcmen, B. A. (2004). Women's characteristics and status. In: Hacettepe University Institute of Population Studies (Hrsg.), Turkey Demographic and Health Survey, 2003. Ankara: Hacattepe University Institute of Population Studies, Ministry of Health, General Directorate of Mother and Child Health and Family Planning, State Planning Organization and European Union, S. 3344.

Hank, K. \& Buber, I. (2007). Grandparents caring for their grandchildren: Findings from the 2004 Survey of Health, Ageing and Retirement in Europe. Mannheim: Research Institute for the Economics of Aging. 
Hans, S. (2010). Assimilation oder Segregation? Anpassungsprozesse von Einwanderern in Deutschland. Wiesbaden: VS Verlag für Sozialwissenschaften (Dissertation).

Heering, L. \& ter Bekke, S. (2008). Labour and income. In: Crul, M. \& Heering, L. (Hrsg.), The position of the Turkish and Moroccan second generation in Amsterdam and Rotterdam. The TIES study in the Netherlands. Amsterdam: AUP, S. 87-103.

Höhne, J., Koopmans, R. (2010). Host-country cultural capital and labor market trajectories of migrants in Germany. Berlin: Wissenschaftszentrum Berlin für Sozialforschung (WZB Discussion Paper, February 2010).

Höpflinger, F. (1993). Weibliche Erwerbsbiographien und Abhängigkeiten zwischen den Generationen. In: Lüscher, K. \& Schultheiß, F. (Hrsg.), Analysen zum Verhältnis von Individuum, Familie, Staat und Gesellschaft. Konstanz: UVK, S. 299-309.

Kalmijn, M. (2006). Educational inequality and family relationships: Influences on contact and proximity. European Sociological Review, 22, 1, 1-16.

Kalmijn, M. \& Saraceno, C. (2008). A comparative perspective on intergenerational support. Responsiveness to parental needs in individualistic familialistic countries. European Societies, 10, 3, S. 479-508.

Kalter, F. (2008). Ethnische Ungleichheit auf dem Arbeitsmarkt. In: Abraham, M. \& Hinz, T. (Hrsg.), Arbeitsmarktsoziologie. Probleme, Theorien, empirische Befunde. Wiesbaden: VS Verlag für Sozialwissenschaften, S. 303-332 (2. Auflage).

Kawaguchi, D. \& Miyazaki, J. (2009). Working mothers and sons' preferences regarding female labor supply: Direct evidence from stated preferences. Journal of Population Economics, 22, S. 115-130.

Lin, N. (1999). Social networks and status attainment. Annual Review of Sociology, 25, S. 467-487.

Long, J. E. (1980). The effect of Americanization on earnings: Some evidence for women. Journal of Political Economy, 88, S. 620-629.

Maliepaard, M., Lubbers, M. \& Gijsberts, M. (2010). Generational differences in ethnic and religious attachment and their interrelation. A study among Muslim minorities in the Netherlands. Ethnic and Racial Studies, 33, 3, S. 451-472.

Milewski, N. (2011). Transition to a first birth among Turkish second-generation migrants in Western Europe. Advances in Life Course Research, 16, 4, S. 178-189.

Nauck, B. (2004). Familienbeziehungen und Sozialintegration von Migranten. Osnabrück: Universität Osnabrück, Institut für Migrationsforschung und Interkulturelle Studien (IMIS-Beiträge 23), S. 83104.

Nauck, B. (2011). Kulturelles und soziales Kapital als Determinante des Bildungserfolgs bei Migranten? In: Becker, R. (Hrsg.), Integration durch Bildung. Wiesbaden: Springer VS, S. 71-93.

Nauck, B. \& Suckow, J. (2006). Intergenerational relationships in cross-cultural comparison: How social networks frame intergenerational relations between mothers and grandmothers in Japan, Korea, China, Indonesia, Israel, Germany, and Turkey. Journal of Family Issues, 27, S. 1159-1185.

Neuwirth, N., \& Wernhart, G. (2007). Die Entscheidung von Müttern zur Erwerbspartizipation. Institutionelle Rahmenbedingungen, Werthaltungen und Aufteilung der Haushaltsarbeit. Wien: Österreichisches Institut für Familienforschung, (Working paper 65).

Olbermann, E. (2003). Soziale Netzwerke, Alter und Migration: Theoretische und empirische Exploration zur sozialen Unterstützung älterer Migranten. Dortmund: Universität Dortmund (Dissertation).

Reher, D. S. (1998). Family ties in western Europe: Persisting contrasts. Population and Development Review, 24, 2, S. 203-234.

Reimers, C. W. (1985). Cultural differences in labor force participation among married women. American Economic Review, 75, 2, S. 251-255.

Rendall, M. S., Tsang, F., Rubin, J. K., Rabinovich, L. \& Janta, B. (2010). Contrasting trajectories of labor-market integration between migrant women in western and southern Europe. European Journal of Population, 26, S. 383-410.

Rumbaut, R.G. (2004). Ages, life stages, and generational cohorts: Decomposing the immigrant first and second generations in the United States. International Migration Review, 38, 3, S 1160-1205.

Seifert, W. (1997). Occupational and economic mobility and social integration of Mediterranean migrants in Germany, European Journal of Population, 13, S. 1-16. 
Söhn, J. (2011). Immigrants' educational attainment: A closer look at the age-at-migration effect. In: Wingens, M., Windzio, M., de Valk, H. \& Aybek, C. (Hrsg.), A life-course perspective on migration and integration. Dordrecht et al.: Springer, S. 27-53.

Soremski, R. (2008). Soziale Mobilitätsorientierung in Familien türkischer Herkunft. Zur Wertetransmission aus der Perspektive der zweiten Generation. In: Hunger, U., Aybek, C. M, Ette, A. \& Michalowski, I. (Hrsg.), Vergemeinschaftung oder nationalstaatliche Lösungswege. Wiesbaden: VS Verlag für Sozialwissenschaften, S. 235-252.

Steinbach, A. \& Nauck, B. (2004). Intergenerationale Transmission von kulturellem Kapital in Migrantenfamilien. Zur Erklärung von ethnischen Unterschieden im deutschen Bildungssystem. Zeitschrift für Erziehungswissenschaft 7, 1, S. 20-32.

Stichs, A. (2008). Arbeitsmarktintegration von Frauen ausländischer Nationalität in Deutschland. Eine vergleichende Analyse über türkische, italienische, griechische und polnische Frauen sowie Frauen aus Nachfolgestaaten des ehemaligen Jugoslawiens. Nürnberg: Bundesamt für Migration und Flüchtlinge (BAMF Working Paper 20).

Sürig, I. \& Wilmes, M. (2011). Die Integration der zweiten Generation in Deutschland. Ergebnisse der TIES-Studie zur türkischen und jugoslawischen Einwanderung. Osnabrück: Universität Osnabrück, Institut für Migrationsforschung und Interkulturelle Studien (IMIS-Beiträge 39).

van Tubergen, F. (2006). Religious affiliation and attendance among immigrants in a secular society. A study of immigrants in the Netherlands. Journal of Ethnic and Migration Studies, 33, 5, S. 747-765.

van Tubergen, F. (2008). The impact of the partner on the economic incorporation of immigrants. Household specialization or social capital? In: Kalter, F. (Hrsg.), Migration und Integration. Wiesbaden: VS Verlag für Sozialwissenschaften (Kölner Zeitschrift für Soziologie und Sozialpsychologie, Sonderheft 48), S. 307-324.

Tucci, I. (2011). National context and logic of social distancing: Children of immigrants in France and Germany. In: Wingens, M., Windzio, M., de Valk, H. \& Aybek, C. (Hrsg.). A life-course perspective on migration and integration. Dordrecht et al.: Springer, S. 143-164.

Tucci, I. \& Yildiz, S. (2012). Das Alterseinkommen von Migrantinnen und Migranten: zur Erklärungskraft von Bildungs- und Erwerbsbiografien. In: Baykara-Krumme, H., Motel-Klingebiel, A. \& P. Schimany, P. (Hrsg.). Viele Welten des Alterns. Ältere Migranten im alternden Deutschland. Wiesbaden: Springer VS, S. 101-126.

Uunk, W., Kalmijn, M. \& Muffels, R. (2005). The impact of young children on women's labour supply: A reassessment of institutional effects in Europe. Acta Sociologica, 48, S. 41-62.

Wengler, A., Schmitt, C. \& Trappe, H. (2008). Partnerschaftliche Arbeitsteilung und Elternschaft. Analysen zur Aufteilung von Hausarbeit und Elternaufgaben auf Basis des Generations and Gender Survey. Wiesbaden: Bundesinstitut für Bevölkerungsforschung

Wheelock, J. \& Jones, K. (2002). "Grandparents are the next best thing": Informal childcare for workings parents in urban Britain. Journal of Social Policy, 31, 3, S. 441-463.

Yavuz, S. (2008). Fertility decline in Turkey from the 1980s onwards: Patterns by main language groups. Ankara: Hacattepe University Institute of Population Studies (Dissertation).

Zimmermann, H.-P. (2012): Altersbilder von türkischen Migrantinnen und Migranten in Deutschland im Vergleich. Islamische Grundsätze - alltägliche Sichtweisen. In: Baykara-Krumme, H., MotelKlingebiel, A. \& Schimany, P. (Hrsg.). Viele Welten des Alterns. Ältere Migranten im alternden Deutschland. Wiesbaden: Springer VS, S. 315-337.

Eingereicht am/Submitted on: 11.08.2012

Angenommen am/Accepted on: 22.02.2013 
Anschrift der Autorin/Address of the author:

Dr. Nadja Milewski

Universität Rostock

Institut für Soziologie und Demographie

Ulmenstr. 69

18057 Rostock

Deutschland/Germany

E-Mail: nadja.milewski@uni-rostock.de 